\title{
A dosimetric approach for relating the biological response of the lung to the accumulation of inhaled mineral dust
}

\author{
J H Vincent, K Donaldson
}

\begin{abstract}
Results from studies of the retention of contrasting mineral dusts inhaled by rats (for periods of up to three months) and the resultant changes in the phagocyte defence system of the deep lung were examined. The dusts used were titanium dioxide (relatively innocuous) and quartz (relatively toxic). The parameters assessed included the accumulation of material in the lung and lymph nodes during chronic exposure and the associated leukocyte response as assessed by bronchoalveolar lavage. The principal findings were that: $(a)$ low level exposure to titanium dioxide produced no measurable inflammation (as indicated by neutrophil recruitment) but higher concentrations $\left(30,50\right.$, and $\left.90 \mathrm{mg} / \mathrm{m}^{3}\right)$ caused the transfer of dust to lymph nodes and first evidence of inflammation; and $(b)$ for quartz, there was a more prominent response and earlier transfer of material to the lymph nodes. The suggested relation between changes in the neutrophil population and dust accumulation is discussed in terms of a quantitative dosimetric model, from which implications for assessing and managing human exposures emerge.
\end{abstract}

The relation between exposure of the respiratory system to harmful dusts and the subsequent development of associated lung disease are both complicated and variable, involving: ( $a$ ) inhalation of particles and their deposition, clearance, and redistribution within the lung; (b) integrated dose of "harmfulness" to lung tissue (where harmfulness represents some property of the particulate material relevant to the potential hazard); (c) biological responses, including inflammation; and $(d)$ tissue damage and repair or destruction. A full description of the disease process requires consideration of all these factors. In this

Institute of Occupational Medicine, Edinburgh EH8 9SU

$\mathrm{J} H$ Vincent, K Donaldson paper we focus attention on some central issues, specifically: $(a)$ the kinetic behaviour of particle transfer within the lung, $(b)$ its relation to a meaningful definition of integrated dose of harmfulness, and $i$ (c) the resultant cellular response.

Dust accumulation and alveolar leukocyte $\mathcal{N}$ response have been investigated recently at the 을 Institute of Occupational Medicine in two separate but complementary studies aimed primarily at $₹$ elucidating these processes for inhaled coalmine dusts. ${ }^{12}$ To gain an appreciation of what happens $\vec{\bullet}$ for non-pathogenic and pathogenic dusts, the $\varnothing$ experiments were carried out using two contrasting insoluble mineral dusts-relatively innocuous titanium dioxide and relatively toxic quartz. The present paper is based on findings derived from bringing together some of the results from these two studies. Full descriptions of the experimental methods used are given in the reports cited and only the essential details will be repeated here.

Deposition, clearance, and redistribution of particles within the lung and associated lymph nodes

Vincent $e t$ al have described experiments in which titanium dioxide (rutile, mass median aerodynamic diameter $1.5 \mu \mathrm{m}$ ) and quartz (Sikron F600, mass median aerodynamic diameter $2.5 \mu \mathrm{m}$ ) were inhaled by SPF rats of the AF/HAN strain for up to 16 weeks (for five days a week, seven hours a day). ${ }^{3}$ Rats were killed in groups of up to four after prescribed periods of exposure and postexposure. Exposure levels for the experiments comprised nominal "respirable" dust concentration ranging widely from 1 to $90 \mathrm{mg} / \mathrm{m}^{3}$ for the titanium dioxide and from $0 \cdot 1$ to $30 \mathrm{mg} / \mathrm{m}^{3}$ for the quartz. The results of the analyses of lung and lymph node burdens were used to construct a pharmacokinetic model (fig 1), the main features of which include:

Deposition of particles into "compartments" from which some may be cleared via the mucociliary escalator.

Transfer of some particles to another "compartment" from which they are not cleared (the "sequestration" compartment).

Lung burden scaling in proportion to the exposure level. 


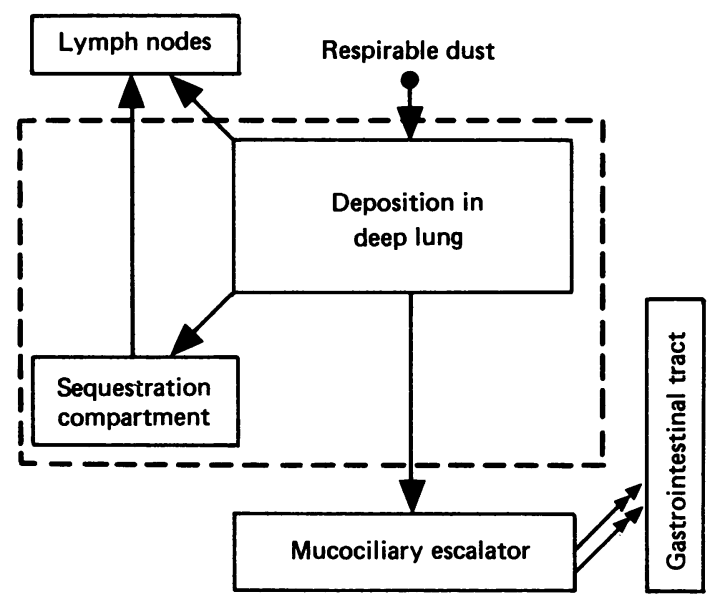

Figure 1 Schematic diagram for pharmacokinetic model describing deposition, clearance, redistribution, and storage of dust within lung.

Transfer of some particles to lung associated lymph nodes, driven by the mass burden in the lung itself but at a rate that does not become significant until the lung burden has exceeded a certain "threshold" value.

Here the term compartment is used to refer to the mathematical features of the lung's response to inhaled particles and not explicitly to its anatomical characteristics, although plausible biological suggestions have been made linking the two in much of the research in this area. It is also worth noting in passing that broadly similar trends had earlier been found in similar experiments with rats inhaling amosite asbestos. ${ }^{45}$

Quantitatively, when this model was first applied to the experimental data and its various numerical coefficients estimated, it was suggested that effective overall clearance of dust out of the lung was broadly comparable for the two dusts. A more recent examination of the data, however, indicates that, in fact, the quartz was cleared substantially more slowly than the titanium dioxide (A M Johnston and $\mathrm{K}$ Donaldson, unpublished data). So far as transport to lymph nodes is concerned, although the actual rates were of the same order for the two materials, the threshold of lung burden at which transfer "took off" was much lower for quartz. This would account for the apparently more rapid transfer to lymph nodes reported by some other workers. ${ }^{6}$

The main features of this model are broadly consistent with those proposed in recent years by other workers based on results for a range of insoluble materials, including not only quartz and titanium dioxide but also diesel particulate ${ }^{7}$ (and S C Soderholm at the EPA diesel emissions symposium, Raleigh, NC, 1981). The same main features also hold for insoluble fibrous dusts such as amosite asbestos. $^{5}$ One aspect that remains uncertain, however, concerns the apparent impairment of clearance ("overload") at high lung burdens, an effect first identified in an earlier paper from this laboratory. ${ }^{4}$ Morrow has drawn attention to the role of the pulmonary macrophage in the overload phenomenon and to how it might vary for different inhaled materials. ${ }^{8}$ Whereas there is indeed strong experimental evidence, from animal inhalation studies, for reduced clearance at high lung burdens during postexposure, ${ }^{46}$ the evidence is less clear for continuous exposures. For example, from the Vincent et al chronic inhalation studies referred to above, ${ }^{3}$ the fact that lung burdens scale in direct proportion to exposure level over such wide ranges reflects the absence of any clear cut consequences that might be expected to be associated with overload. On the other hand, from the results of longer term inhalation studies with rats reported by Wolff $e t$ al for rats exposed to diesel particulate, ${ }^{9}$ an overload effect begins to become apparent at high concentrations and for exposure times beyond one year. Elsewhere, in inhalation studies involving toner particulate (as used commercially in the photoreproduction process) the effect is even more pronounced.$^{10}$ Clearly, for overload, there remain several unanswered questions, including the suggestion that different kinetics might be required to account for what happens during continuous exposure and postexposure respectively.

\section{Cellular response to particles in the lung}

From a parallel programme of experiments carried out in our laboratory, Donaldson et al have reported results for the corresponding cellular responses associated with the inhalation of dust. ${ }^{2}$ These inhalation studies used the same test dusts and basic exposure regimens as those described above, at nominal respirable dust concentrations of 10 and 50 $\mathrm{mg} / \mathrm{m}^{3}$. One difference worthy of note is that, here, the animals used were syngeneic PVG rats bred under specific pathogen free conditions, but this is not expected to influence substantially the particular conclusions we shall be attempting to draw by combining the two sets of results.

Samples recovered from the rat lungs by bronchoalveolar lavage were assayed to provide a wide range of biological parameters. In this paper, however, attention will be focused on just one indicator of the lung's initial biological response to the dust insult - the accumulation of neutrophils in the bronchoalveolar space. It is a reasonable first assumption that this, being a quantitative measure of the level of inflammation, is an appropriate index relevant to subsequent biological processes leading ultimately to diseases such as pneumoconiosis and emphysema. 
For given periods of exposure and postexposure, the numbers of neutrophils recovered by bronchoalveolar lavage were determined. The results are presented in figs 1 and 2, where the neutrophil numbers are plotted as a function of time elapsed since exposure started. The circular symbols refer to animals killed immediately at the termination of exposure, the diamonds to animals killed at the stated time postexposure. They show that, for the titanium dioxide, cell counts for exposure at $10 \mathrm{mg} / \mathrm{m}^{3}$ did not significantly differ from zero. At $50 \mathrm{mg} / \mathrm{m}^{3}$, however, a sudden rise in neutrophil numbers occurred between 72 and 105 days since the start of exposure. In the 62 day postexposure phase following 105 days of exposure the count subsequently fell to about half of its end of exposure value. For the quartz, the magnitude of the response was much greater. Even at $10 \mathrm{mg} / \mathrm{m}^{3}$, there were significant numbers of neutrophils for exposure times beyond about 40 days. At 50 $\mathrm{mg} / \mathrm{m}^{3}$, the onset of recruitment occurred even earlier, at about 10 days. After onset, the neutrophil counts increased steadily during continuing exposure. Most interestingly, however, and in pronounced contrast to the results for titanium dioxide, the numbers went on increasing after exposure had ceased.

\section{Cellular response in relation to particle dose}

To begin with, it is relevant to consider the relation between the onset of the cellular response and the onset of lymphatic clearance. From the experiments referred to earlier, Vincent $e t$ al estimated the lung burden at which clearance to lymph nodes "took off" for titanium dioxide and quartz (1800 and $900 \mu \mathrm{g}$ respectively). ${ }^{3}$ From the pharmacokinetic model

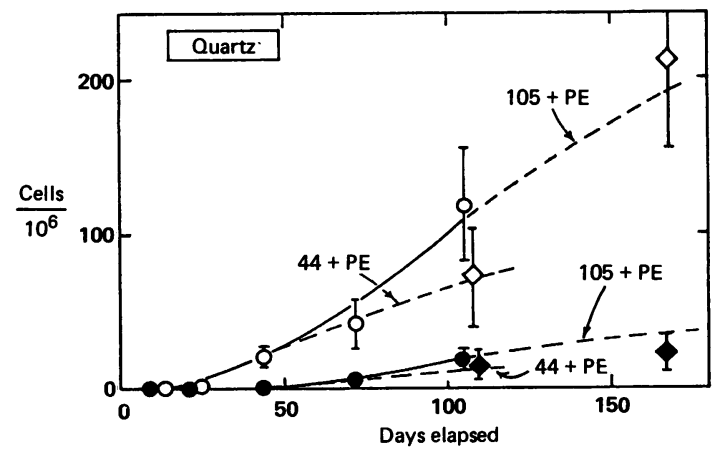

Figure 2 Neutrophil numbers as a function of time elapsed for quartz dust at exposure concentrations of 10 and $50 \mathrm{mg} / \mathrm{m}^{3}$ (solid and open symbols, respectively). Circles refer to animals killed immediately at end of exposure and diamonds to those killed at indicated time postexposure. Error bars define experimental uncertainty. Curves relate to neutrophil numbers predicted by dosimetric model, under working assumptions stated in text; solid lines refer to continuous exposure, broken lines to postexposure beyond exposure period indicated-for instance, $105+P E)$. derived from the same body of work, the lung burden at which, in the cell studies, the neutrophil count $\overrightarrow{\vec{B}}$ began to rise significantly may also be estimated. For $\frac{?}{\circ}$

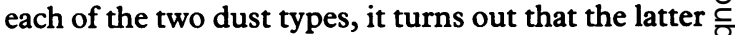
threshold value is of the same order to magnitude as that for the onset of lymphatic clearance. From such estimates, therefore, it would appear that the two $ᄋ$ types of response are closely linked.

We now set out to examine the nature of integrated $\vec{\circ}$ dose, as a means of relating the cellular response of the lung to the long term accumulation of particulate $\vec{\omega}$ material. Here, the contrasting responses to titanium $\bigcirc$ dioxide and quartz, especially postexposure, provide $\frac{\Phi}{3}$ valuable insight into how to proceed. Qualitatively, the results show that the response to titanium dioxide of falls postexposure by an amount that is greater pro $\dot{\omega}$ rata than the estimated fall in lung burden due to $\mathrm{N}$ clearance. On the other hand, for quartz, the 을 response carries on increasing even though we know that the the lung burden cannot increase further. It is $\bar{z}$ therefore clear that the instantaneous burden of material in the lung is not, on its own, a meaningful $\vec{\theta}$ descriptor of the biological outcome.

There are three essential components of a dosimetric hypothesis on which to base the assessment of risk associated with inhaling airborne particles. The first is the exposure history, which o may be expressed in terms of the exposure function, $E_{n}$. This is the time weighted average exposure $\propto$ concentration (or intensity) during the $\mathrm{n}^{\text {th }}$ day since $\overrightarrow{\overrightarrow{0}}$ the start of exposure. The second component is the 3 time dependent retention of particles in the lung, where $R_{m}$, the retention function, is the proportion remaining of a particular "packet") of material at the end of the $m^{\text {th }}$ day since it was deposited (where $m$ can $\stackrel{\infty}{3}$ take all values up to and including $n$ ). This may be $\dot{0}$ based on pharmacokinetic models such as the one 3 shown in fig 1. The third component relates to the $\delta$ ability of the particles to transmit "harmfulness" to tissue. For a given material at the end of the $\mathrm{m}^{\text {th }}$ day 0 since a packet has been deposited, this is expressed as $\mathrm{G}_{\mathrm{m}}$. This may be referred to as the harmfulness function and is a function of the particulate material itself and of the time since the particle arrived in the $\sigma$ lung. Thus it embodies both the instantaneous $N$ potency of the material (which in turn is responsible $\mathrm{N}_{\mathrm{N}}$ for driving the corresponding instantaneous 0 biological response) and its persistence.

To illustrate the concept of cumulative dose based $\stackrel{\bullet}{\odot}$ on these concepts, consider the simple case of three of days of exposure followed by two days postexposure. For this we may construct the array

$\begin{array}{ll}\text { Day } & \text { Incremental dose received } \\ 1 & E_{1} R_{1} G_{1} \\ 2 & E_{1} R_{2} G_{2}+E_{2} R_{1} G_{1} \\ 3 & E_{1} R_{3} G_{3}+E_{2} R_{2} G_{2}+E_{3} R_{1} G_{1} \\ 4 & E_{1} R_{4} G_{4}+E_{2} R_{3} G_{3}+E_{3} R_{2} G_{2} \\ 5 & E_{1} R_{5} G_{5}+E_{2} R_{4} G_{4}+E_{3} R_{3} G_{3}\end{array}$


where the overall cumulative dose is the sum of all these individual contributions. In general, for $\mathrm{N}$ days of exposure and $\Delta \mathrm{N}$ days of postexposure, this is given by

$$
D_{N, \Delta}=\sum_{n=1}^{N+\Delta N} E_{n} \sum_{m=1}^{N+\Delta N-n+1} R_{m} G_{m}
$$

where, for all $n>N, E_{n}$ obviously must be zero. It should be noted that, mathematically, this solution is not strictly rigorous (N A Esmen, personal communication). This stems from the fact that $E$, by virtue of the way in which it must be measured and recorded, is a time averaged, and hence discrete, quantity. On the other hand, both $R$ and $G$ are mathematically continuous functions. It is therefore possible to envisage problems in using equation (1) in certain extreme situations-for example with rapidly varying exposure levels coupled with comparably rapid changes in pharmacokinetic response. Hence equation (1) may not be entirely general. Nevertheless, it may be shown to be adequate for computational purposes in potential applications of dosimetry in relation to long term health effects associated with insoluble particulate matter.

To link all this to the actual biological outcome, we need a working hypothesis linking integrated dose and response. Perhaps the simplest starting point is to assume that: $(a)$ the relevant biological response parameter is the neutrophil population; $(b)$ this is directly proportional to integrated dose of harmfulness, and $(c)$ the rate at which the particulate material stimulates neutrophil influx does not change with the time the material spends in the lung-that is, $G$ is constant. Based on the comments made at the beginning of this section about the role of lymphatic clearance, a further qualifying assumption is justified-namely, that effective dose only starts to accumulate once the lung burden has passed the threshold for which transfer of material to lymph nodes takes off.

In relating the dosimetric model to the experimental animal inhalation data, it may be taken that $\mathrm{E}$ is constant (at 10 or $50 \mathrm{mg} / \mathrm{m}^{3}$ ), as this is a reasonably good approximation of the experimental conditions that prevailed. $R$ as a function of time is derived from the pharmacokinetic model in fig 1 , using the coefficients for quartz and titanium dioxide as determined by Vincent $e t a l{ }^{3}$ The results are shown in fig 2 for quartz, where experimental points represent the experimental results for neutrophil numbers and the drawn curves represent predicted numbers values (based on the working assumptions stated above). The solid curves represent what happens during continuous exposure (for the two exposure levels) and the broken curves represent

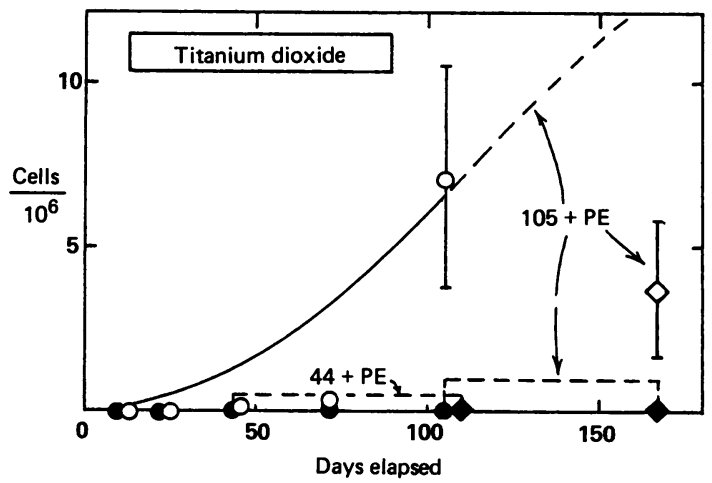

Figure 3 Neutrophil numbers as function of time elapsed for titanium dioxide at exposure concentrations of 10 and $50 \mathrm{mg} / \mathrm{m}^{3}$. (Legend same as for figure 2).

what happens during postexposure. Figure 2 exhibits quite good agreement between experiment and theory for a single fitted value of the harmfulness function, $G$ (=110, in arbitrary units), for the two contrasting exposure levels $\left(10\right.$ and $\left.50 \mathrm{mg} / \mathrm{m}^{3}\right)$, and for both the exposure and the postexposure phases. Thus we have confirmation that, in biological terms, this material is highly persistent. This means that harmfulness continues to be delivered to the lung for as long as the particles are in contact with the tissue. There is apparently no decay in potency-nor, indeed, any increase.

The results for titanium dioxide are less easy to interpret. As shown in fig 3, whereas we can choose a value of $\mathrm{G}(=5$, much smaller than for quartz) that gives rough agreement for the exposure phase, the simple set of starting assumptions cannot account for the fall in response postexposure. A modification so that $G$ is a rapidly decaying function of time would improve the situation somewhat in that it would restrict the continued rise in integrated dose. This would seem to be plausible for titanium dioxide in the light of its well known innocuousness. This single adjustment is not, on its own, sufficient to explain the fall in neutrophil counts. It would appear necessary also to build in negative contributions arising from the lung's ability to recover from the insult and return to normalcy. This too seems plausible. Unfortunately, at this stage, there are insufficient experimental data to enable us to develop and quantitatively test such a hypothesis. This therefore is an important area for further work.

\section{Discussion \\ FINDINGS FROM THE RESEARCH}

The principal aim of this paper has been to link changes in the patterns of the build up of mineral dusts in the lung and associated lymph nodes with concomitant changes in the alveolar neutrophil population. 
The first, but not entirely surprising, observation is that the two dusts exhibit a remarkable difference in their estimated initial magnitudes for the harmfulness function $(G)$, reflecting their relative abilities to deliver harmfulness to lung tissue- 110 for quartz compared with only five for titanium dioxide. This appears to be one major factor in the differences in ability to cause lung injury and inflammation since the other likely factors (such as exposure intensity and the rates of deposition and clearance) are broadly similar for the two dusts. It is particularly interesting that quartz continues to deliver injury to the lung (as reflected in the continuing increase in neutrophil population) even during the postexposure period. By contrast, the corresponding postexposure response for titanium dioxide actually declines. A plausible explanation for this is that titanium dioxide is not persistent in its potency and that the lung is able to recover relatively rapidly from this particular insult. This therefore confirms what is already well known, that quartz presents the greater potential problem.

\section{COMPARISON WITH OTHER DUSTS}

Our findings may be compared with those derived from similar experiments carried out in our laboratory to investigate cellular responses in rats exposed to coaline dusts. ${ }^{2}$ Broadly, the overall results show that, so far as inflammation is concerned, the magnitude of the response may be ranked:

titanium dioxide $<$ coalmine dusts $<$ quartz.

On the other hand, the ability of the lung to recover postexposure may be ranked in reverse order, relating to the magnitude of the inflammation present at the end of the exposure period:

quartz $<$ coalmine dusts $<$ titanium dioxide.

More specifically, lungs exposed to titanium dioxide exhibited sharply decreasing inflammation postexposure, lungs exposed to coalmine dusts showed persistence of inflammation but no further progression, and, as we have seen, lungs exposed to quartz showed pronounced progression.

\section{IMPLICATIONS TO OCCUPATIONAL DUST RELATED LUNG DISEASE}

Applying this whole scenario to workers exposed to such dusts, then the implications for occupational health may be highly significant, especially in relation to workers who have left an industry where they have been exposed to quartz. From the sequestration hypothesis, it is reasonable to deduce that for a man who has spent a lifetime working in such an industry, the vast majority of the dust particles present in his lung will have been sequestrated and so will not be available for clearance. This, together with the evidence that quartz continues to deliver considerable levels of harmfulness to the lung postexposure, may be a clue to understanding the progressive

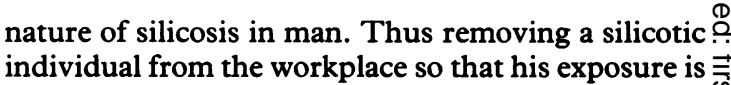
curtailed may not prevent progression of the disease $\stackrel{?}{?}$ and the development of subsequent disease. This is consistent with what has been reported epidemio- $\frac{\bar{D}}{\bar{D}}$ logically. ${ }^{11}$ The problem is brought out particularly $\frac{\text { Tn }}{\sigma}$ clearly in the results described in this paper, opening $\propto$ the door to the possibility that quantitative predic- is tion of progression in workers exposed to quartz $\vec{\circ}$ might be achieved.

The observation that the onset of inflammation is $\overrightarrow{\vec{\omega}}$ dependent on the exposure intensity is important in $\frac{\Omega}{\circ}$ relation to the establishment of occupational $\frac{\Phi}{3}$ exposure limits, since such inflammation is generally accepted as being a precursor to pathological changes in the lung tissue. The fact that inflammation of occurred in response to titanium dioxide albeit only ${ }_{N}$ significantly at higher exposure levels, suggests that 0 no dust should be considered as totally innocuous so long as a sufficient quantity can be deposited in the $\vec{z}$ lung. Recently, Lee et al have shown, in experiments with rats, that pathological change can indeed be induced by chronic exposure to high airborne 8 concentrations of titanium dioxide. ${ }^{12}$ So the widely held view that dusts such as titanium dioxide are "inert" needs to be treated with caution. The methods described here provide the opportunity to quantify the hazard associated with such dusts.

Finally, the above findings may provide some $\stackrel{\varnothing}{\perp}$ useful ideas for epidemiological research into dust $\overrightarrow{\vec{B}}$ related health effects. The integrated dose of $\stackrel{3}{\exists}$ harmfulness as expressed in equation (1) differs considerably from the cumulative exposure index.

$$
C_{N}=\sum_{n=1}^{N} E_{n}
$$

which has been the foundation of much previous epidemiological inquiry. Equation (2) is convenient 0 since it does not require any knowledge of, or imply? any importance to, the time dependent shape of the exposure history. But, on the evidence of our findings presented in this paper, equation (1) would $\sigma$ appear in general to be the more appropriate. To N apply equation (1) raises the difficulty of how to $\mathrm{N}$ quantify both $\mathrm{E}$ (the time weighted average exposure concentration) and $\mathrm{G}$ (the harmfulness function) in a manner relevant to the human exposure situation. At? present we have only a preliminary grasp of the problem based on experiments with animals. So substantial further work is required before the desired goal can actually be achieved. In the mean- $\mathbb{\mathbb { D }}$ time, it is interesting to note that equation (1) $\frac{\mathrm{P}}{\mathrm{Q}}$ becomes equivalent to equation (2) for particulate $\varrho$ materials that are either cleared rapidly from the lung or, as in the case of titanium dioxide, rapidly become 8 innocuous after they have been deposited. For coal? 
dust which, as we have seen, provides a range of responses somewhere between that for titanium dioxide and quartz, it may be possible to argue that equation (2) is adequate. But we do not believe this to be the case for quartz.

\section{Conclusions}

The study was based on the assumption that bronchoalveolar lavage neutrophil populations are a general measure of tissue injury and inflammation. We have related these populations to the dose of harmfulness delivered to the lungs of rats for inhaled airborne dusts of contrasting type and known ability to produce disease-namely, quartz and titanium dioxide. The dosimetric estimates were based on a mathematical model for describing the kinetics of the retention of dust in the lung and pulmonary lymph nodes. The main conclusions are that:

(1) Inhalation of quartz, known to be a relatively toxic dust, gives rise to a substantial level of inflammation that is highly persistent and progresses even after exposure has ceased. The latter is associated with the long term retention and sequestration of a material whose potency to stimulate injury remains high throughout its residency in the lung.

(2) Inhalation of sufficiently large quantities of even a relatively non-toxic dust such as titanium dioxide dust can elicit a short term inflammatory response. This suggests the possibility that pathological changes may ensue if exposure to any inert dust is long enough and intense enough. The magnitude of such a response, however, is much less than for more toxic dusts such as quartz. Furthermore, it is much less persistent, decaying rapidly postexposure.

(3) The development of inflammation, for both types of dust, is closely associated with transfer of dust to the lymph nodes and this could be an important factor in the pathological changes that occur in the lungs after chronic inflammation, in particular fibrosis and emphysema.

This picture points to the importance of determining whether improved exposure management, based on a proper appreciation of the kinetics of the various biological processes governing the true integrated dose to tissue, can further reduce the likelihood of the development of lung disease. Furthermore, acceptance of the importance of the role of the inflammatory response in the aetiology of dust related lung fibrotic diseases suggests the possibility of a test for individual susceptibility. Clearly much further work is required and justified.

We thank the many colleagues at the Institute of Occupational Medicine for their contributions to this research and also the Commission of European Communities and British Coal Corporation for their financial support.

1 Jones AD, Vincent JH, McMillan $\mathrm{CH}$, et al. Animal studies to investigate the deposition and clearance of inhaled mineral dusts. (Final report on CEC contract 7248/33/026.) Edinburgh: Institute of Occupational Medicine, 1988. (IOM report $\mathrm{TM} / 88 / 05$.)

2 Donaldson K, Bolton RE, Brown DM, et al. Studies on the cellular response in lung tissue to the inhalation of mineral dust. (Final report on CEC contract 7248/33/025.) Edinburgh: Institute of Occupational Medicine, 1988. (IOM report $\mathrm{TM} / 88 / 01$.)

3 Vincent JH, Jones AD, Johnston AM, McMillan C, Bolton RE, Cowie $\mathrm{H}$. Accumulation of inhaled mineral dust in the lung and associated lymph nodes: implications for exposure and dose in occupational lung disease. Ann Occup Hyg 1987; 31:375-93.

4 Bolton RE, Vincent JH, Jones AD, Addison J, Beckett ST. An overload hypothesis for pulmonary clearance of UICC amosite fibres inhaled by rats. $\mathrm{Br} J$ Ind $M e d$ 1983;40:264-72.

5 Vincent JH, Johnston AM, Jones AD, Bolton RE, Addison J. Kinetics of deposition and clearance of inhaled mineral dusts during chronic exposure. $\mathrm{Br} J$ Ind Med 1985;42:707-15.

6 Klosterkotter W, Bunemann G. Animal experiments on the elimination of inhaled dust. In: Davies CN, ed. Inhaled particles and vapours. Oxford: Pergamon Press, 1961:327-37.

7 Strom KA, Chan TL, Johnston JT. Pulmonary retention of inhaled sub-micron particles in rats: diesel exhaust exposures and lung retention model. Warren (MI): General Motors Research Laboratories, 1987. (Res publ GMR-5718.)

8 Morrow PE. Possible mechanisms to explain dust overloading of the lungs. Fundam Appl Toxicol 1988;10:369-84.

9 Wolff RK, Henderson RF, Snipes MB, et al. Alterations in particle accumulation and clearance in lungs of rats chronically exposed to diesel exhaust. Fundam Appl Toxicol 1987; 9:154-66.

10 Muhle $\mathrm{H}$, Bellmann B, Creutzenberg $\mathrm{O}$, et al. Pulmonary deposition, clearance and retention of test toner, titanium dioxide and quartz during a long-term inhalation study in rats. Toxicologist 1988;8:270.

11 Morgan WKC, Seaton A. Occupational lung diseases. Philadelphia: WB Saunders, 1984.

12 Lee KP, Trachimowicz HJ, Reinhardt CF. Pulmonary response of rats exposed to titanium dioxide (TiO2) by inhalation for two years. Toxicol Appl Pharmacol 1985;79:179-92.

Accepted 16 October 1989 